Supporting Information for:

\title{
Nanoporous Gold Membranes as Robust Constructs for Selectively Tunable Chemical Transport
}

\author{
Daniel A. McCurry and Ryan C. Bailey* \\ Department of Chemistry, University of Illinois at Urbana-Champaign, 600 S. Mathews Ave., Urbana, \\ Illinois, 61801, USA \\ *baileyrc@illinois.edu
}

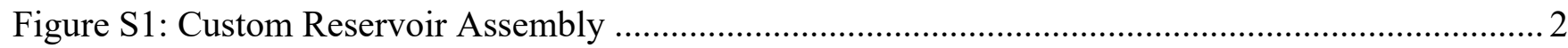

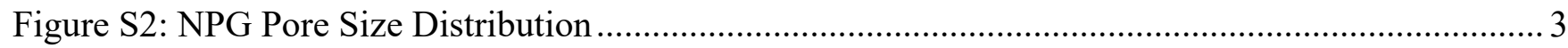

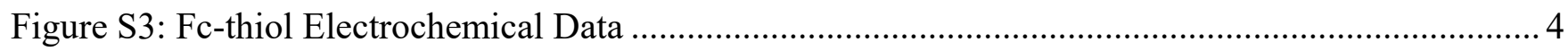

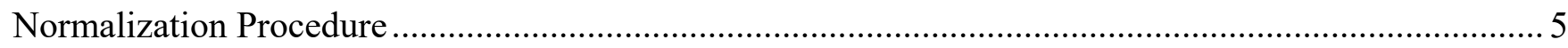

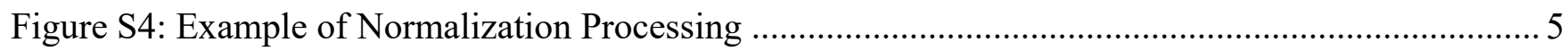


(a)

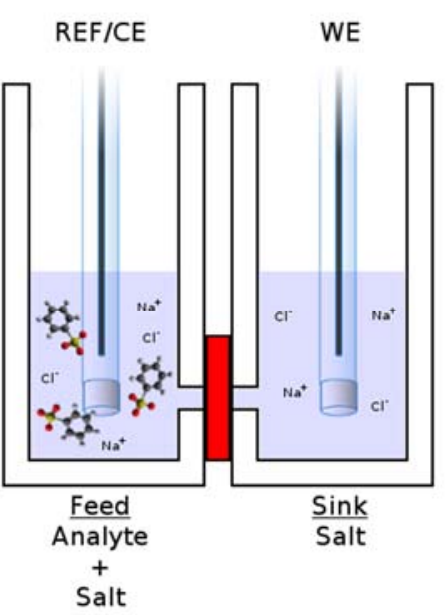

(b)

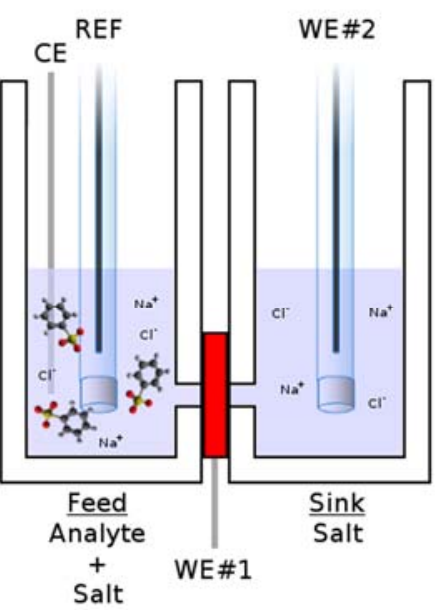

(c)

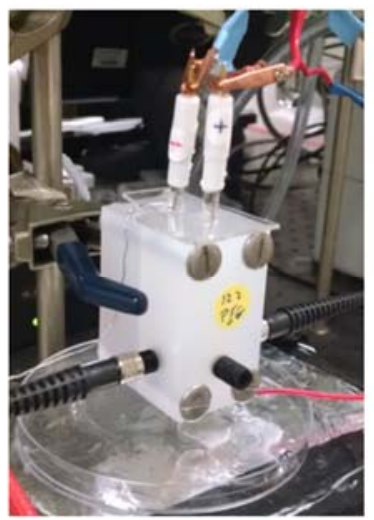

Figure S1: Custom Reservoir Assembly

(a) Schematic of reservoir assembly used for $\mathrm{pH}$ sensitive thiol coated NPG. (b) Schematic of reservoir assembly for NPG with direct applied potential. Note the addition of a Pt CE to the feed reservoir. This was required to account for the additional current from the large surface area of the NPG. (c) Photograph of the custom reservoir assembly. The NPG is sandwiched between the two reservoirs. A fiberoptically coupled UV-visible spectrometer measured flux in real time as potentials were applied across and to the NPG. 


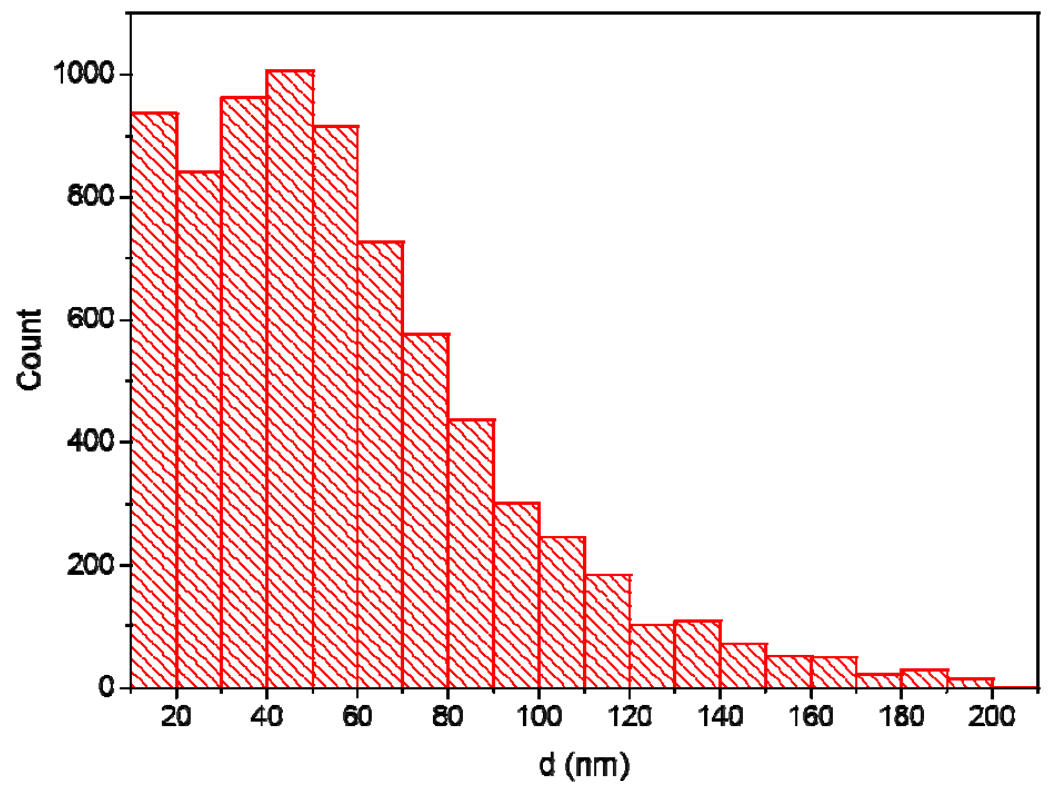

\section{Figure S2: NPG Pore Size Distribution}

Measured pore diameters using automated pore thresholding in ImageJ. A median of $50 \pm 20 \mathrm{~nm}$ pore diameters are reported. This presents a fairly large distribution of pore sizes which should be advantageous for future size-selective separations. 
(a)

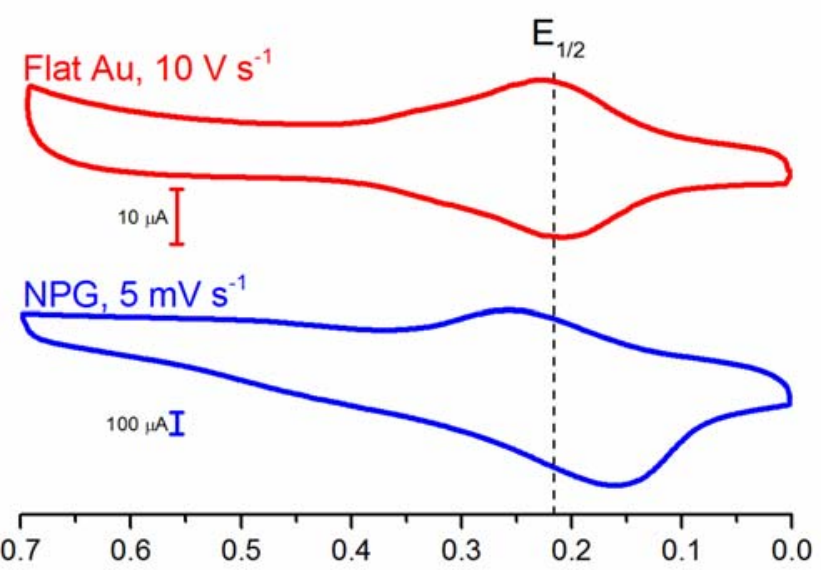

(b)

$E(V)$
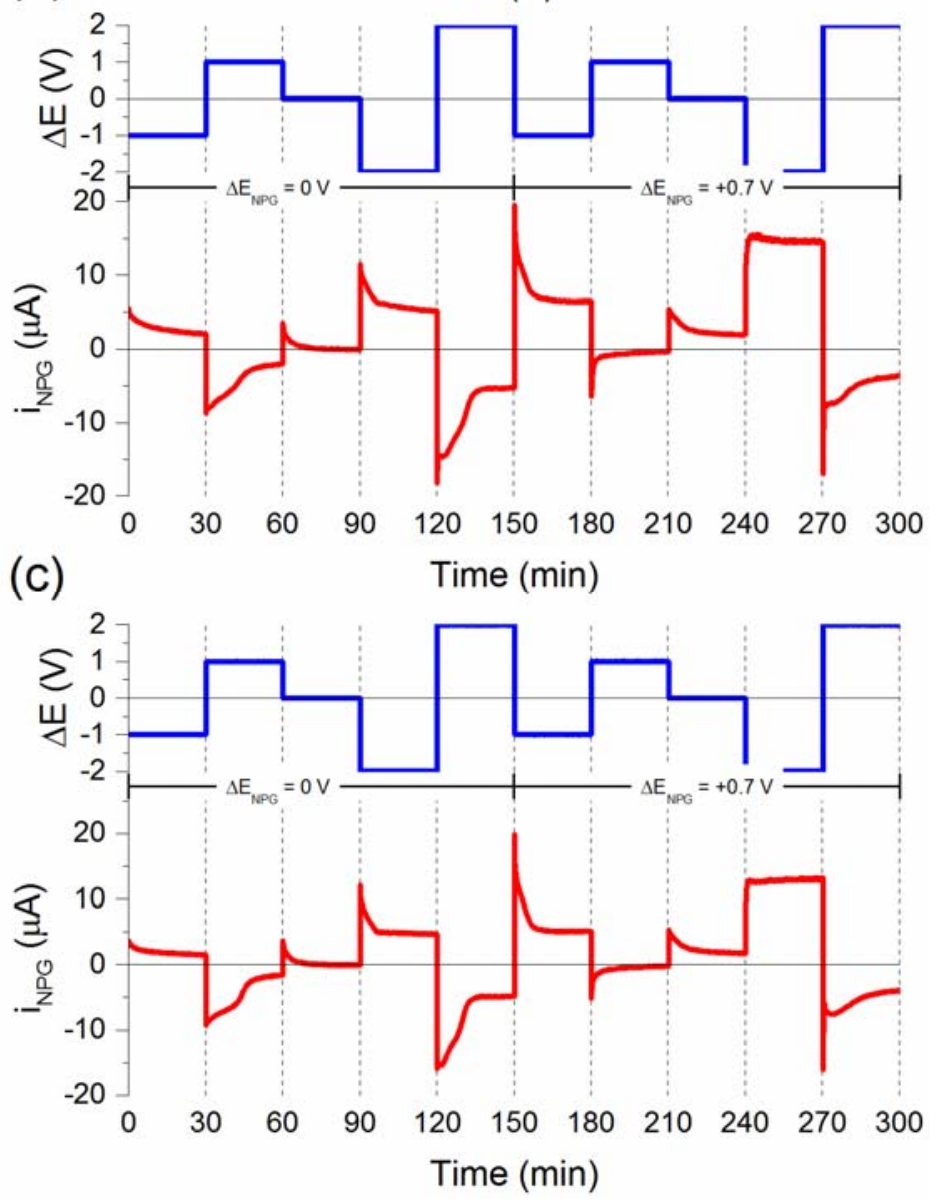

Figure S3: Fc-thiol Electrochemical Data

(a) Cyclic voltammogram of the Fc-terminated thiol on both flat Au and NPG in $0.1 \mathrm{M}$ sodium perchlorate. The thiol was deposited in a 1:22 ratio of 6-mercapto-1-hexanol to 6-ferrocenyl-1-hexanethiol and the scan rates were adjusted to clearly resolve the peaks. The E1/2 should ideally be equal to the $E_{p a}$ and $\mathrm{E}_{\mathrm{pc}}$ for a surface adsorbed electroactive species. The peak deviation and non-faradaic charging increases for the NPG due to the close proximity of the ferrocene groups and very large surface area, respectively. The current measured at the NPG (red) during application of a transverse potential (blue) for (b) methyl viologen and (c) benzene sulfonate demonstrates large currents at the NPG for a significant period of time. These currents may contribute to the measured flux. 


\section{Normalization Procedure}

Due to the intricate geometry of each NPG sample, flux through the pores is sample dependent. The nonlinear effect of altering pore size (electroosmotic contributions and the ratio of EDL to pore volume) complicates direct comparison. In order to accurately compare experimental results, the molecular flux measured for each run was averaged to obtain an average flux. The datasets were then normalized to one another according to:

$$
j^{\prime}=j \frac{j_{\text {ref mean }}}{j_{\text {mean }}}
$$

where $j^{\prime}$ is the normalized flux at a value of $\Delta \mathrm{E}, j$ is the non-normalized flux at a value of $\Delta \mathrm{E}, j$ ref mean is the flux of the reference averaged dataset, and $j_{\text {mean }}$ is the flux of the individual averaged dataset. This allows for each dataset to be normalized to arbitrary molecular flux values and subsequently averaged, correcting for NPG samples with different baseline translocation rates. Division of the average values at $\Delta \mathrm{E} \neq 0$ by $\Delta \mathrm{E}=0$ permits comparison of flux relative to the flux at $\Delta \mathrm{E}=0$, or in other words, the transport enhancement factor with applied potential relative to passive diffusion.
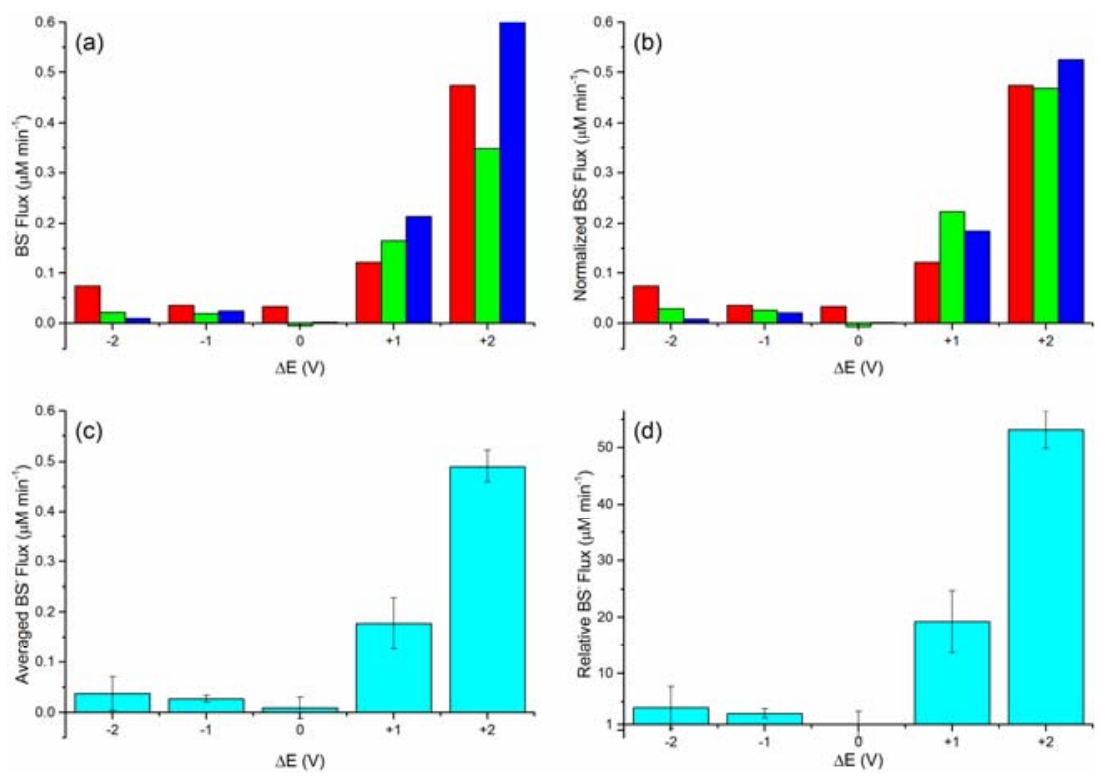

\section{Figure S4: Example of Normalization Processing}

(a) $\mathrm{BS}^{-}$flux through amine-thiol-functionalized NPG at acidic $\mathrm{pH}$ as measured from the real-time slope of absorbance vs. time from three runs. Qualitatively, all three runs demonstrate similar trends of increased translocation rate at more positive potentials. (b) Normalization of the molecular fluxes to the average flux of the first run (red). The second run (green) had a lower average flux than the first run and was therefore normalized to have the same average flux. Similarly, the average flux of the third run was normalized. (c) Average normalized flux from all three runs. (d) Flux relative to the flux at $\Delta \mathrm{E}=0$. Note that the $\mathrm{x}$-intercept has changed from 0 to 1 